\title{
Wh-questions in the Trinidad and Tobago Signing Community
}

\author{
Felicia Bisnath ${ }^{1}$ \\ University of Michigan, Ann Arbor
}

\section{Introduction}

Signing in Trinidad and Tobago is characterised by variation and multilingualism. These properties arise out of the community's history of deaf education, which began in the early 1940s. In 1975, there was a switch in pedagogical approach from Oralist methods to Total Communication, which introduced contact with American Sign Language (ASL). These two periods are associated with two signing varieties: Trinidad and Tobago Sign Language (TTSL) with the former and Trinidad and Tobago ASL (TTASL) with the latter. The nature of the grammatical differences between these varieties has not been described yet, and they are known, used and mixed by different kinds of signers in different contexts. Additionally, they may both be subsumed under the term "TTSL" by some signers.

This paper describes variation in the domain of wh-questions as they are used by three different kinds of consultants: two deaf people who grew up with and without deaf parents and relatives respectively, and a hearing person with deaf parents. Whquestions have not been described in Trinidad and Tobago signing yet and are typologically valuable because their syntax appears to be a site of modality-based difference. Describing their use in Trinidad and Tobago adds a typological datapoint, sheds some light on the nature of variation in Trinidad and Tobago signing and begins to identify similarities and differences between TTSL and TTASL.

The structure of this paper is the following: section 2 describes signing in Trinidad and Tobago, section 3 details the properties of $w h$-questions in signed languages, section 4 describes the research design and language consultants, section 5 is a description of how wh-questions are used in the Trinidad and Tobago signing community, section 6 situates $w h$-questions in Trinidad and Tobago signing in the typological literature and identifies similarities and differences between TTSL and TTASL, and section 7 concludes the paper with suggestions for future research.

\section{Signing in Trinidad and Tobago}

Signing in Trinidad and Tobago occurs in a multilingual context and is subject to language contact and variation. This situation is a product of the history of deaf education in Trinidad and Tobago and of the hearing community that TTSL is embedded in. Braithwaite (2018) identifies 2 varieties, that differ in age and the level of contact they have had with ASL: TTSL which emerged at a deaf school

\footnotetext{
${ }^{1}$ This paper would not have been possible without Roland Pfau (University of Amsterdam), Ben Braithwaite (University of the West Indies, St. Augustine, Trinidad and Tobago) and the deaf and hearing language consultants: Ian Dhanoolal, Joel Jaggernauth and Bryan Rodrigues.
} 
from 1946-75 with minimal influence from ASL, and TTASL which emerged as a local dialect of ASL starting in 1975. Throughout this paper, I use the terms TTSL and TTASL in the ways defined above, but it is important to note that grammatical distinctions between the varieties have not been described and may not be salient to all users.

\subsection{Deaf education and signed language emergence in Trinidad and Tobago}

The first deaf school in Trinidad, the Cascade School for the Deaf, was opened in 1943 in Trinidad by a deaf signer of British Sign Language (BSL). In 1946, it was moved to another location and began to accept boarders in addition to day students. From 1946-75, oralist methods were used. Such methods aim to teach deaf children how to speak and lipread, rely on amplification methods, and dissuade the use of manual communication. Despite this, it has been suggested that a north American manual alphabet was in use at the time and some BSL could have been present too (Braithwaite, 2018). During this time, TTSL was developed by deaf children during their breaks from the classroom and in their dormitories. In 1975, oralist methods were replaced by Total Communication. Total Communication does not emphasise spoken language use, but encourages the use of all communicative resources available, such as the hands and body. With the advent of Total Communication, ASL and Signing Exact English II (SEE-II) were introduced into deaf education. This had two consequences: (i) it altered the development of TTSL since signed languages with more social value were present where they were not before and (ii) it began the development of a local variety of ASL, TTASL.

Currently, there are 2 deaf schools in Trinidad (the second opened in 1971). Deaf students are also taught alongside hearing students in mainstreaming programmes that began in 1980 and are accommodated in special education and vocational schools (Parks \& Parks, 2012). Apart from formal education, various religious bodies teach signed languages - some teach ASL and some a combination of ASL and what they call TTSL. People also learn how to sign informally by interacting with the deaf community.

\subsection{TTSL vs TTASL}

The primary distinction this paper makes between TTSL and TTASL is that they are associated with different time periods and levels of contact with spoken and signed languages. TTSL began developing first with minimal influence from signed languages and formal influence from Trinidadian English/Creole via oralism. When ASL and signed English were introduced, the development of TTSL was interrupted and TTASL began to develop. Because of this temporal difference, not all signers are aware of what I refer to as the TTSL variety and so do not make a distinction between TTSL and TTASL. Researchers pre-dating Braithwaite (2018) also do not make a distinction between TTSL and TTASL, and are often referring to the later variety, TTASL, when they use "TTSL". 
Little is known about TTSL and TTASL and how and if they are different. Parks \& Parks (2012) describe TTASL as "something that looks like Signed English with heavy initialization of signs" (9), and Braithwaite (2018) calls it a local variety of ASL. There is evidence of lexical differences and differences in non-manual marking (hereafter NMM) - where ASL uses the mouthing "cha" to mean large, TTSL uses "pow" (Braithwaite 2018). Regarding syntax, TTSL is SVO with the possibility of SOV order (Kwok 2015). Methodologically, the lack of a clear distinction between TTSL and TTASL among signers and in linguistic research means that any study of signing in Trinidad and Tobago must be cognisant of the social and educational background of consultants. This approach is also generally relevant when conducting research in urban deaf communities due to the heterogeneous nature of signed language acquisition.

\section{Wh-questions in signed languages}

Wh-questions, also called content questions are those that ask for a specific type of information, as opposed to polar questions which only require affirmation or negation of a proposition. Typologically, they are valuable since they can be assumed to be found in all languages (Mackenzie 2009: 1133). When describing them in signed languages, three components are relevant: NMMs, the inventory of $w h$-words (the $w h$-paradigm) and the position of the $w h$-word. I briefly discuss each of these in turn, beginning with NMM since it is found at both the lexical and syntactic levels.

\subsection{Non-manual marking}

Non-manual markers are articulations of parts of the face (eyebrows, eyes, mouth, lips, cheeks etc.) that accompany manual signing to identify clause type and information structure, disambiguate the meanings of nouns, and modify nouns and verbs. In $w h$-questions in signed languages, they function at the syntactic and lexical levels. In this section, I discuss the form of $w h$-NMMs in the syntax and lexicon to serve as background for the more detailed discussions of these levels in sections 3.2 and 3.3 respectively.

In syntax, they are used to mark question type. In western signed languages, the form of the wh-NMM is similar, involving furrowing of the eyebrows (Zeshan 2006: 41). Wh-NMMs are typically articulated simultaneously with the manual signs for $w h$-words; however, they can occur on their own, as in Japanese Sign Language (Nihon Shuwa). Wh-NMM can spread to parts of the clause other than the $w h$-word and in some signed languages, like Italian Sign Language (LIS), this has been analysed as marking a syntactic dependency (Cecchetto et al. 2009) (see section 3.3 for more).

In the lexicon, wh-NMM takes the form of mouthings, which are defined as movements of the mouth that are related to spoken language words (Nadolske \& 
Rosenstock, 2007: 40). Wh-mouthings accompany manual wh-words and can either have the same meaning as the manual sign (e.g. the spoken language word for what mouthed while making the manual sign for what) as in ASL, or can disambiguate the meanings of general manual question signs (e.g. the spoken language word for what mouthed while making the manual sign for a general interrogative) as in Yolngu Sign Language (YSL) (Australia) (Bauer 2014: 110)and Israeli Sign Language (ISL) (Meir 2004).

\subsection{Wh-paradigms}

The $w h$-paradigm in a language is its inventory of $w h$-words, which typically corresponds to English who, what, when, where, why, which, how, and how many/much. In signed languages, there is often a general interrogative corresponding to "what" that covers the wh-paradigm to different extents. Zeshan (2006) uses the role of this general interrogative to identify different kinds of paradigms:

(i) the general interrogative accounts for the entire $w h$-paradigm e.g. InSL

(ii) the general interrogative accounts for part of the wh-paradigm e.g. Brazilian Sign Language (LIBRAS), Nihon Shuwa

(iii) the general interrogative exists in addition to idiosyncratic signs for all wh-words e.g. ASL

Wh-questions can also include question particles as in Finnish Sign Language (FinSL), though this is rare (Zeshan 2006: 61). They are not obligatory, but when used, they mark sentence type and occur in the same prosodic unit as the question. They should be considered when describing $w h$-paradigms since their form can resemble that of "what" and/or a general interrogative.

\subsection{Syntax}

In the literature on signed languages, interrogatives have been described in New Zealand, Japanese, Turkish, American, Hong Kong, Flemish, Brazilian, Finnish, Indo-Pakistani (in Zeshan 2006), Israeli (Meir 2004) Providence Island (Washabaugh, Woodward \& DeSantis 1978), Ban Khor (Nonanka 2010), and

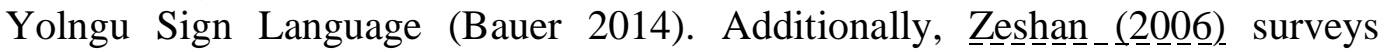
interrogatives across 35 signed languages. In a smaller number of signed languages wh-movement analyses have been made (see Abner (2011), Petronio \& LilloMartin (1997) and Neidle et al. (1998) on ASL, Cecchetto, Geraci \& Zucchi (2009) and Branchini et al. (2013) on Italian Sign Language; Aboh, Pfau \& Zeshan (2005) on Indo-Pakistani Sign Language and Lillo-Martin \& de Quadros (2006) on ASL and Brazilian Sign Language).

These studies have shown that the position of the $w h$-word in signed languages is not as restricted as it is in spoken languages. The $w h$-word can be found clauseinitially, clause-finally and duplicated clause-initially and -finally in signed 
languages. Based on the clause-final and duplicated data, analyses of rightward movement have been proposed in ASL (Neidle et al. 1998) and Italian Sign Language (LIS) (Cecchetto, Geraci \& Zucchi 2009); however, the case of ASL is not clear-cut, with Petronio \& Lillo-Martin (1997) arguing for leftward movement. Some of the issues in the ASL analyses arise from disagreement over judgements. Abner (2011) also shows that differences in the semantics of $w h$-questions can be linked to differences in syntax. In contrast to this, of the 1200 spoken languages listed in the World Atlas of Language Structures (WALS), only one shows the option for rightward movement, Tenet (South Sudan) (Dryer 2013). Additionally, there is "no attested case of a sign language that would allow only clause-initial interrogatives (Zeshan 2006: 64). Based on these patterns, it has been suggested that the syntax of $w h$-questions may be a locus of macro-typological variation due to modality (Cecchetto, Geraci \& Zucchi 2009).

In this paper, I refer to $w h$-movement because it is important in the literature on $w h$ questions; however, I do not make any strong claims about it in Trinidad and Tobago signing because doing so requires information about other parts of the grammar that are not yet known.

\section{Research Design}

An integrated approach to data collection was taken in this study to compensate as much as possible for the weaknesses of individual tasks (Van Herreweghe \& Vermeerbergen 2012). Four tasks were concatenated: formal elicitation, informal elicitation, grammaticality judgements and interview. The elicitation was conducted by Ben Braithwaite of the University of the West Indies, St. Augustine, in Trinidad, and grammaticality judgements and interviews were conducted remotely, with Ben Braithwaite interpreting. As for my positionality, I am a hearing researcher who, at the time of this study, had not been living in Trinidad and Tobago for a year and had not had extensive experience working with deaf researchers and signed languages, or using signed languages. At the time of the writing of this paper, I am more experienced in these areas, but I would consider myself more of an outsider than an insider to the deaf community in Trinidad and Tobago, and therefore, I bring the biases associated with that position to this paper. In the following sections, I describe the language consultants (section 4.1) and the methods (4.2).

\subsection{Consultants}

The language consultants in this study represent some of heterogeneity found in the deaf community in Trinidad and Tobago. Signed language communities like that found in Trinidad and Tobago are characteristically heterogeneous because in them, deaf children are typically born to hearing parents who do not use a signed language. This means that signed language acquisition does not proceed in the same way for all deaf children and can be impacted by how parents choose to educate their children and their perspectives on the use of cochlear implants. The 
consultants in this study represent differences in deaf status, age, signed language acquisition path, language background and educational background. They are similar in that they are all active members of the deaf community in Trinidad and Tobago and have served in leadership roles and were not part of the founding TTSL cohorts. In this section, I describe the consultants further (see table 1 for a summary).

Consultant 1 is a deaf man born to hearing parents; therefore, he was not exposed to signing until he began to attend school. He went to the Cascade School when Total Communication was in use where he received input from signed English and ASL. He also learned TTSL from custodial staff who were part of the founding cohorts. After this, he spent a year in the United States at a deaf high school where he was further exposed to ASL. Currently, he works as a tutor at the University of the West Indies in Trinidad and Tobago where he teaches TTSL/TTASL. He is also involved in linguistics research and has done fieldwork with signing communities in Guyana, the Bay Islands, San Andreas and Providence Island. Consultant 2 is a hearing man born to deaf parents and learned to sign from them as a child. Because he is hearing, he attended a mainstream hearing school and was not formally taught a signed language. He is also a native speaker of Trinidad English/Creole. Consultant 3 is a deaf man born to deaf parents and has deaf siblings and relatives. Some of these relatives were part of the founding TTSL cohorts, which means that he was exposed to the language since he was born.

\begin{tabular}{|c|c|c|c|c|c|}
\hline Consultant & Age & Status & Job & Education & Languages \\
1 & 43 & Deaf & Teacher/Researcher & CSD & TTSL, \\
& & & & TTASL \\
2 & 23 & Hearing & Interpreter & Hearing & TTASL, \\
& & CODA & school & TE/C \\
& 38 & $\begin{array}{c}\text { Deaf } \\
\text { CODA }\end{array}$ & Salesperson & CSD & TTSL \\
\hline
\end{tabular}

Table 1: Characteristics of language consultants

$(\mathrm{CSD}=$ Cascade School for the Deaf; $\mathrm{CODA}=$ child of deaf adults; $\mathrm{TE} / \mathrm{C}=$ Trinidadian English/Creole)

\subsection{Methods}

The following methods were applied in an order that gradually revealed what study was about i.e. the formal elicitation task was done first, followed by informal elicitation, grammaticality judgements and interview. This was done to collect as much information as possible before explicitly asking about the structure and use of $w h$-questions.

The formal elicitation task is a novel picture-based approach, based on Geraci et al. (2015) and Friedmann \& Szterman (2011). A hybrid task was developed to ensure 
that there were prompts for the entire $w h$-paradigm, and to collect enough data per consultant. The task is done in pairs, with one consultant holding a complete picture of a scene (Consultant A) (see figure 1, left), and the second holding the same scene but with part of it blocked out (Consultant B) (see figure 1, right). Consultant B must ask Consultant $\mathrm{A}$ a question to figure out what is missing from their scene. Each consultant was shown 2 stimuli designed to elicit who, what, when, where, why, which, how (instrument) and how many/how much i.e. each consultant was shown 16 stimuli.

05

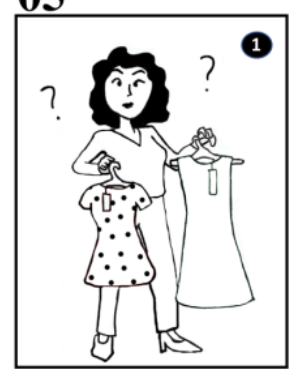

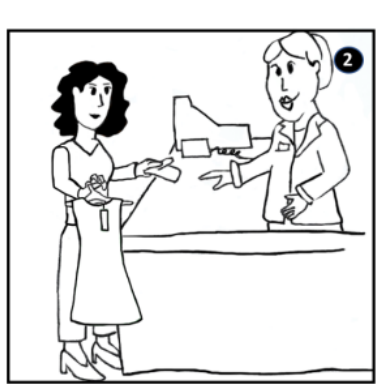

05

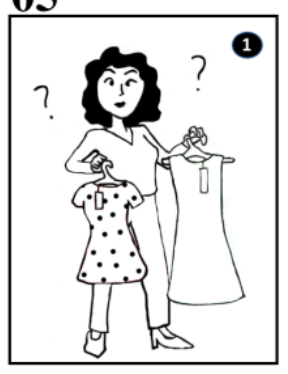

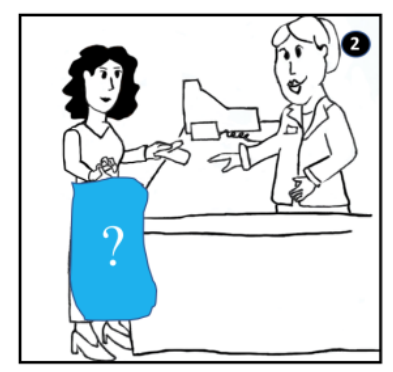

Figure 1: Prompts for "which" (Left panel = Consultant A, Right panel = Consultant B)

After the formal task, the consultants were explicitly asked for signs for wh-words that did not emerge during elicitation. In this session, I took on the role of someone learning to sign and the consultants were asked to show at least 2 examples of questions using those signs. This was necessary because the stimuli were not equally successful at eliciting individual $w h$-words, and it is not always necessary to use an idiosyncratic $w h$-word to ask a $w h$-question.

After elicitation, grammaticality judgements and individual interviews were conducted. In the grammaticality judgments, the same sentences, with $w h$-words in different positions, were presented to the consultants. The sentences were first shown individually, and then as a group, and consultants were asked to say which sentence they preferred and why.

\section{Description of wh-questions in Trinidad and Tobago Signing}

In this section, I describe the form of the wh-paradigms and wh-NMM used in the Trinidad and Tobago signing community, and present preliminary data on the scope of $w h$-NMM and the syntax of $w h$-questions. In general, consultants 1 and 2 tended to pattern with each other, while consultant 3 patterned differently.

\subsection{Wh-words}

Eighteen unique signs for $w h$-words were produced by the 3 language consultants. All these signs are produced with the corresponding Trinidadian English/Creole mouthings. These signs can be divided into two paradigms - one that resembles ASL, that I assign to TTASL (see figure 3), and another that I assign to TTSL (see 
figure 2). The TTASL paradigm was produced by consultants 1 and 2 and the TTSL paradigm by consultant 3 . I made the division in the way described because (i) consultant 3 grew up around people who were involved in the creation of TTSL (ii) consultant 3 explicitly identified the signs produced by consultants 1 and 2 as belonging to ASL and (iii) the TTASL signs resembled ASL signs.

The TTASL paradigm consists of 11 signs and is phonologically almost identical to the current ASL paradigm, apart from (i) the use of older signs for who (WHOTTASL2) and what (WHAT-TTASL2) alongside the modern varieties, and (ii) phonological differences in the production of why and (what) time. In ASL, why is formed using the Y-handshape and is articulated at the side of the cheek while in TTASL, the sign begins at the forehead and uses the B-handshape oriented toward the body and ends with the Y-handshape in neutral space in front of the body. In TTASL, (what) time is articulated while mouthing "what time", while in ASL no mouthing is used.

The TTSL paradigm consists of 8 signs. Seven of these signs use a flat B-handshape oriented upwards (who, what, when, where, why, how, how much). Of these 7, all are primarily distinguished by NMMs such as mouthings and movements of the body. The exception to this is who, which has the index finger pointed to the mouth initially. Where is accompanied with side-to-side head movements, and why is accompanied by forward movement of the body, which was also observed when consultants 1 and 2 produced why. The one sign that does not use the flat Bhandshape is (what) time and is also found in TTASL.

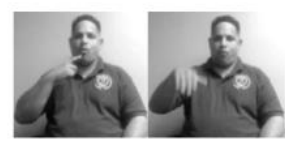

a. WHO-TTSL

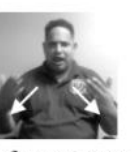

f. WHY-TTSL

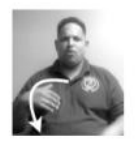

b. WHAT-TTSL

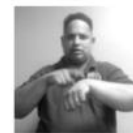

g. (WHAT) TIME-TTSL

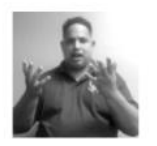

c. WHEN-TTSL

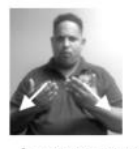

h. HOW-TTSL
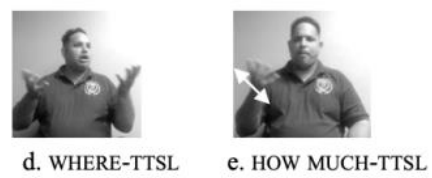

Figure 2: TTSL Paradigm 

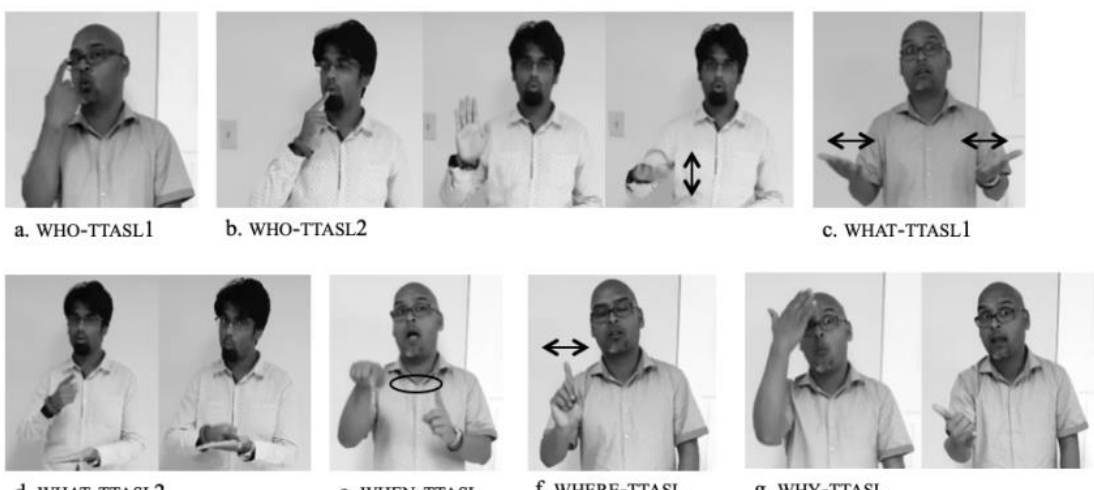

e. WHEN-TTASL

f. WHERE-TTASL

g. WHY-TTASL

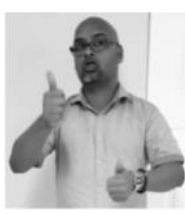

h. WHICH-TTASL

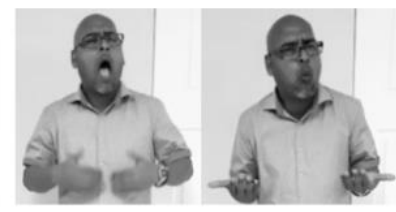

i. HOW-TTASL

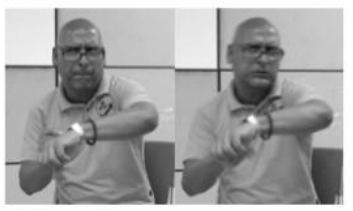

k. (WHAT)TIME

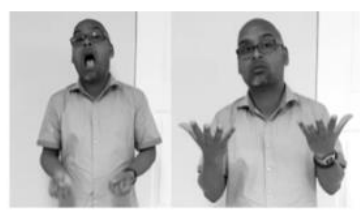

j. HOW.MUCH-TTASL

Figure 3: TTASL Paradigm

\subsection{Non-manual marking}

Two patterns of NMM were observed across all consultants (see figure $4 \mathrm{a}$ and c) when producing $w h$-questions. The $w h$-question pattern (wh), is a combination of furrowed eyebrows + squinted eyes (figure $4 \mathrm{a}$ ) and is may combine with a raised chin (figure $4 b$ ). The second pattern is raised eyebrows + widened eyes (figure 4c) and appears to be used to mark topics.

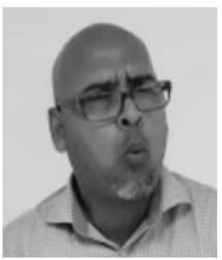

a. wh

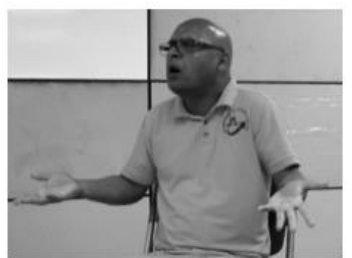

b. wh + chin up

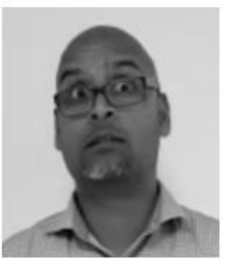

c. $t p$

Figure 4: Non-manual marking patterns

Wh-NMM can occur independently of $w h$-words, and as shown in examples $\mathrm{X}$ and $\mathrm{X}$, they can be used to form $w h$-questions on their own; however, this strategy is 
not productive as shown by example (2). This suggests that example (1) is idiomatic. For example (2) to be grammatical, a wh-word must be mouthed (in this case, which), which suggests that manual wh-signs are not the only kinds of $w h$ words available. However, example (2) is constructed and it is not known how productive this strategy is. My impression is that it is not common.

(1)

$$
\begin{aligned}
& \overline{\text { old }}^{\text {wh }} \\
& \text { old } \\
& \text { 'How old (are you)?' }
\end{aligned}
$$

(Consultant 3)

(2)

$$
\begin{aligned}
& \text { *boy } \\
& \text { boy } \\
& \text { 'Which boy?' }
\end{aligned}
$$

(Consultant 1)

In $w h$-questions involving manual $w h$-words, $w h$-NMM is always present on $w h$ words. When the $w h$-word is clause-initial or clause-final, wh-NMM was observed on the $w h$-word only or spread across the entire sentence, regardless of if the subject or object is questioned. In other words, spreading does not seem to differ based on the position of the wh-word if movement is assumed or not; however, this study was not designed to capture nuances in spreading.

\subsection{Positions of the wh-word}

The wh-word can be found clause-initially (3) and clause-finally alone (4) and duplicated i.e.in clause-initial and clause-final position (5). Consultants 1 and 2 produced $w h$-words in all positions, while consultant 3 primarily produced $w h$ words clause-finally. Consultant 2 tended to duplicate $w h$-words. Despite the variation in production by consultants 1 and 2, they both agreed with consultant 3 that questions with the wh-word in clause-final position are best. Consultant 3's intuition was that sentences with the $w h$-word in clause-initial position functioned like answers or rhetorical questions. Examples like (4) and (6) respectively suggest that rightward and leftward movement could be possible; however, rightward movement could only be observed with who-questions, assuming SVO order.

$\begin{array}{lll} & & \\ \text { who-TTASL } & \text { eat } & \text { fish } \\ \text { who } & \text { eat } & \text { fish }\end{array}$

'Who ate fish?' 
(4)

$\begin{array}{lll}\text { fish } & \text { eat } & \text { who-TTASL } \\ \text { fish } & \text { eat } & \text { who }\end{array}$

'Who ate fish?'

(Consultant 1)

(5)

\begin{tabular}{llll}
\hline why-TTASL & girl & cry & why-TTASL \\
why & girl & cry & why
\end{tabular}

'Why is the girl crying?'

(Consultant 2)

(6)

$\begin{array}{lll} & & \\ \text { where-TTASL } & \text { girl } & \text { hide } \\ \text { where } & \text { girl } & \text { hide }\end{array}$

'Where is the girl hiding?'

(Consultant 2)

\section{Discussion}

Wh-questions in Trinidad and Tobago are associated with eighteen unique whwords, one NMM form and four positions of the $w h$-word. These grammatical properties when viewed alongside the backgrounds of the language consultants (refer to section 4.1) reveal variation between TTSL and TTASL that has not been described before. In section 6.1, this variation is explained and situated typologically and in section 6.2 I discuss the term "TTSL".

\subsection{Situating wh-questions in Trinidad and Tobago signing}

The clearest locus of difference between TTSL and TTASL occurs in the lexicon, and are also reported by a deaf consultant in the literature:

“At my school, Cascade School, we didn't use American Sign

Language, we only used Trinidadian Sign Language. It was completely different. For example, there were difference [sic] signs for 'mother', 'father', 'woman', 'bus' and 'home'." (Braithwaite 2018: 6)

The $w h$-words can be divided into two paradigms corresponding to the descriptions of TTSL and TTASL in section 2.2. The TTASL paradigm is almost identical to 
the current ASL paradigm but has two additional variants that resemble older ASL signs. Both paradigms have idiosyncratic signs for each wh-word; however, this idiosyncrasy is represented in different ways - in TTSL, handshapes are not as differentiated as in TTASL and disambiguation seems to rely primarily on mouthings, as it does in New Zealand Sign Language (NZSL) (McKee 2006) and Yolngu Sign Language (YSL) (Bauer 2014). The case of NZSL parallels that of Trinidad and Tobago in that there are two paradigms that developed one after the other in response to changes in deaf education and language contact, and that the older paradigm uses a palm up handshape that resembles the co-speech gesture used with questions (Cooperrider, Abner \& Goldin-Meadow 2018) and is disambiguated by mouthings (McKee 2006: 77).

Compared to the lexicon, $w h$-NMM and syntax are more aligned. The form of $w h$ NMM is the same, and is also found in Western signed languages, including ASL and NZSL (Zeshan 2006). However, a clear pattern of $w h$-NMM influenced by the presence or absence of $w h$-movement as described in Italian Sign Language (LIS) (Cecchetto, Geraci \& Zucchi 2009) was not observed. Clear inter-speaker variation in the use of $w h$-NMM was not observed either. Possible reasons for this are that the methods were not fine-grained enough or, assuming movement, $w h$-NMM is not used to mark $w h$-dependency.

In the syntax, all orders described in the literature are found in Trinidad and Tobago signing. The preference for $w h$-words in clause-final position is also found in NZSL and Israeli Sign Language (ISL) (Meir 2004; McKee 2006: 73). In NZSL, whwords are not often found clause-initially but clause-finally and duplicated. In ISL, clause-initial and -final order is allowed, but the latter is considered better and the variation that occurs is attributed to discourse. However, the overarching preference for clause-final $w h$-words may be due to prescriptivism if inter-speaker variation in production is to be accounted for. Prescriptive ASL grammars teach that the whword should be clause-final (Kate Mesh, personal communication, 23 ${ }^{\text {rd }}$ July 2018) - given consultant 1's experience of attending a deaf school in the United States, and consultant 2's job as an interpreter, it is not unlikely that they could have been influenced by prescriptive ASL rules, particularly when asked for metalinguistic information. Consultant 3 could also have been influenced by ASL rules either passively or actively, but given his family, language background, and his focus on distinguishing ASL from TTSL, it seems more likely that in his variety of TTSL (and by extension his family's), the $w h$-word is clause-final. Regarding duplication, it was consultant 3 's view that this configuration was not a question form at all, but was rhetorical, or could be used to answer questions.

\subsection{The name "TTSL" and methodology}

In this paper, I imposed the names TTSL and TTASL on the signing that was observed based on (Braithwaite 2018), inter-speaker variation and metalinguistic observations provided by the consultant most familiar with the older variety of 
signing in Trinidad and Tobago (consultant 3). These names reflect different times and kinds of language contact and can be viewed as the poles of a linguistic continuum. During data collection, the consultants were told that the study was about TTSL - implicitly, they were asked to produce TTSL, but this was not emphasised. The differences in production described in this paper show that different kinds of signers have different ideas of what the term "TTSL" refers to, at least in terms of the lexicon. This variation in terminology is linked to the language background of signers - only those signers who have knowledge of a second named variety can contrast it with other varieties. The major methodological implication coming out of this is that, when studying communities like those found in Trinidad and Tobago and New Zealand where there is a linguistic continuum associated with different approaches to deaf education, it is necessary to connect linguistic practice to signers' backgrounds. This is because the named varieties created by linguists out of convenience do not necessarily reflect the perceptions of all members of a community.

\section{Conclusion}

This paper makes three contributions. First, it sets a descriptive foundation for further study of $w h$-questions in Trinidad and Tobago signing varieties by identifying $w h$-words, $w h$-NMM and positions of the $w h$-word. Second, it identifies grammatical similarities and differences between TTSL and TTASL. Third, it shows that the meaning of the term "TTSL" varies based on a signer's background. Future research on wh-questions would benefit from more fine-grained investigation of the spread of $w h$-NMM, the semantics of $w h$-questions with $w h$ words in different positions and the interaction between the position of the wh-word and NMM.

\section{References}

Abner, Natasha. 2011. WH-words That Go Bump in the Right. In Mary Washburn (ed.), Proceedings of the 28th West Coast Conference on Formal

Linguistics, 24-32. Sommerville, MA: Cascadilla Proceedings Project.

Aboh, Enoch O., Roland Pfau \& Ulrike Zeshan. 2005. When a Wh-word is not a Wh-word: The Case of Indian Sign Language. In Rajendra Singh (ed.), The Yearbook of South Asian Languages and Linguistics (2005). Berlin, New York: Walter de Gruyter. https://doi.org/10.1515/9783110186185.11. http://www.degruyter.com/view/books/9783110186185/9783110186185.1 1/9783110186185.11.xml (13 June, 2018).

Bauer, Anastasia. 2014. The use of signing space in a shared sign language of Australia (Sign Language Typology Series 5). Boston/Berlin: De Gruyter Mouton.

Braithwaite, Ben. 2018. Language Contact and the History of Sign Language in Trinidad and Tobago. Sign Language Studies 19. 5-39. https://doi.org/10.1353/sls.2018.0024. 
Branchini, Chiara, Anna Cardinaletti, Carlo Cecchetto, Caterina Donati \& Carlo Geraci. 2013. wh-duplication in Italian Sign Language (LIS). Sign

Language \& Linguistics 16(2). 157-188. https://doi.org/10.1075/sll.16.2.03bra. http://www.jbeplatform.com/content/journals/10.1075/s1l.16.2.03bra (13 June, 2018).

Cecchetto, Carlo, Carlo Geraci \& Sandro Zucchi. 2009. Another Way to Mark Syntactic Dependencies: The Case for Right-Peripheral Specifiers in Sign Languages. Language 85(2). 278-320. http://www.jstor.org/stable/40492869.

Cooperrider, Kensy, Natasha Abner \& Susan Goldin-Meadow. 2018. The palmup puzzle- meanings and origins of a widespread form in gesture and sign.pdf. Frontiers in Communication 3(23). 1-16. https://doi.org/doi: 10.3389/fcomm.2018.00023.

Dryer, Matthew S. 2013. Position of interrogative phrases in content questions. In Matthew S. Dryer \& Martin Haspelmath (eds.), The World Atlas of Language Structures Online. Leipzig: Max Planck Institute for Evolutionary Anthropology. https://wals.info/chapter/93 (5 September, 2020).

Friedmann, N. \& R. Szterman. 2011. The Comprehension and Production of WhQuestions in Deaf and Hard-of-Hearing Children. Journal of Deaf Studies and Deaf Education 16(2). 212-235.

https://doi.org/10.1093/deafed/enq052.

https://academic.oup.com/jdsde/article-lookup/doi/10.1093/deafed/enq052 (13 June, 2018).

Geraci, Carlo, Robert Bayley, Anna Cardinaletti, Carlo Cecchetto \& Caterina Donati. 2015. Variation in Italian Sign Language (LIS): The case of whsigns. Linguistics 53(1). https://doi.org/10.1515/ling-2014-0031. https://www.degruyter.com/view/j/ling.2015.53.issue-1/ling-20140031/ling-2014-0031.xml (13 June, 2018).

Kwok, Lily. 2015. Investigating constituent order in Trinidad and Tobago Sign Language (TTSL). Trinidad and Tobago: University of the West Indies, St. Augustine BA.

Lillo-Martin, Diane \& Ronice Müller de Quadros. 2006. The Position of Early WH-Elements in American Sign Language and Brazilian Sign Language. In K.U. Deen, J. Nomura, B. Schulz \& B. D. Schwartz (eds.), Proceedings of the Inaugural Conference on Generative Approaches to Language Acquisition -- North America (GALANA), vol. 1, 195-203. Hawai.

McKee, Rachel. 2006. Aspects of interrogatives and negation in New Zealand Sign Language. In Ulrike Zeshan (ed.), Interrogative and negative constructions in sign languages (Sign Language Typology Series 1), 7090. Nijmegen: Ishara Press.

Meir, Irit. 2004. Question and Negation in Israeli Sign Language. Sign Language \& Linguistics 7(2). 97-124. https://doi.org/10.1075/s1l.7.2.03mei. 
http://www.jbe-platform.com/content/journals/10.1075/sll.7.2.03mei (22 July, 2020).

Neidle, Carol, Dawn MacLaughlin, Robert G Lee, Benjamin Bahan \& Judy Kegl. 1998. Wh-Questions in ASL: A Case for Rightward Movement.

Neidle, Carol, Dawn MacLaughlin, Robert G. Lee, Benjamin Bahan \& Judy Kegl. 1998. The Rightward Analysis of wh-Movement in ASL: A Reply to Petronio and Lillo-Martin. Language 74(4). 819. https://doi.org/10.2307/417004. https://www.jstor.org/stable/417004?origin=crossref (18 August, 2018).

Nonanka, Angela M. 2010. Interrogatives in Ban Khor Sign Language. In Gaurav Mathur \& Donna Jo Napoli (eds.), Deaf around the World, 194-220. Oxford University Press. https://doi.org/10.1093/acprof:oso/9780199732548.001.0001. http://www.oxfordscholarship.com/view/10.1093/acprof:oso/9780199732 548.001.0001/acprof-9780199732548 (13 June, 2018).

Petronio, Karen \& Diane Lillo-Martin. 1997. WH-Movement and the Position of Spec-CP: Evidence from American Sign Language. Language 73(1). 18. https://doi.org/10.2307/416592. https://www.jstor.org/stable/416592?origin=crossref (13 June, 2018).

Van Herreweghe, Mieke \& Myriam Vermeerbergen. 2012. Data Collection. In Roland Pfau, Markus Steinbach \& Bencie Woll (eds.), Sign Lanuage: an International Handbook, 1023-1044. Berlin: De Gruyter Mouton.

Washabaugh, William, James C. Woodward \& Susan DeSantis. 1978. Providence Island Sign: A Context-Dependent Language. Anthropological Linguistics 20(3). 95-109. http://www.jstor.org/stable/30027404.

Zeshan, Ulrike (ed.). 2006. Interrogative and negative constructions in sign languages (Sign Language Typology Series 1). Nijmegen: Ishara Press. 